\begin{tabular}{|c|l|}
\hline Title & Local probing of thermal properties at submicron depths with megahertz photothermal vibrations \\
\hline Author(s) & Tomoda, M.; Shiraishi, N.; Kolosov, O. V.; W right, O. B. \\
\hline Citation & $\begin{array}{l}\text { A pplied Physics Letters, 82(4), 622-624 } \\
\text { https://doi.org/40.1063/1539906 }\end{array}$ \\
\hline Issue Date & $2003-01-27$ \\
\hline Doc URL & http://hdl.handle.net/2115/28033 \\
\hline Rights & Copyright $\odot 2003$ A merican Institute of Physics. \\
\hline Type & article \\
\hline File Information & APL82-4.pdf \\
\hline
\end{tabular}

Instructions for use 


\title{
Local probing of thermal properties at submicron depths with megahertz photothermal vibrations
}

\author{
M. Tomoda and N. Shiraishi \\ Department of Applied Physics, Faculty of Engineering, Hokkaido University, Sapporo 060-8628, Japan \\ O. V. Kolosova) \\ Department of Materials, University of Oxford, Parks Road, Oxford, OX1 3PH, U.K. \\ O. B. Wright ${ }^{\text {b) }}$ \\ Department of Applied Physics, Faculty of Engineering, Hokkaido University, Sapporo 060-8628, Japan
}

(Received 26 August 2002; accepted 2 December 2002)

\begin{abstract}
We demonstrate the imaging of buried features in a microstructure- - tiny hole in an aluminum thin film covered by a chromium layer - with nanometer lateral resolution using a transient temperature distribution restricted to within $\sim 0.5 \mu \mathrm{m}$ of the sample surface. This is achieved by mapping photothermally induced megahertz surface vibrations in an atomic force microscope. Local thermal probing with megahertz-frequency thermal waves is thus shown to be a viable method for imaging subsurface thermal features at submicron depths. (C) 2003 American Institute of Physics.
\end{abstract}

[DOI: $10.1063 / 1.1539906]$

The scanning thermal microscope, making use of a probe tip composed of an ultrasmall temperature sensor or resistive heater, has allowed the local imaging of thermal properties on lateral length scales down to $\sim 25 \mathrm{~nm} .{ }^{1,2}$ Of particular interest in this field are techniques in which a transient temperature distribution is used. Thermal waves are sensitive to the heat capacity as well as the thermal conductivity, providing an extra source of contrast. Moreover, unlike the case for varying elastic fields, the intrinsically damped thermal wave propagation can be controlled through the oscillation frequency $f$ : the thermal penetration depthessentially the thermal diffusion length-decreases with increasing frequency as $1 / f^{1 / 2}$, and so it is advantageous in microscopy with thermal waves to work at high frequencies in order to achieve a high spatial resolution. Because of the ongoing trend toward device miniaturization with features less than $\sim 100 \mathrm{~nm}$ in size, local probing of thermal modulation phenomena is an area of paramount importance.

One approach that combines oscillating (that is, ac) temperature fields and local probing is based on the use of a modulated heat source in the tip. ${ }^{3,4}$ Although useful images sensitive to thermal diffusivity have been obtained, such experiments are difficult to interpret because the depth resolution is often strongly influenced by near-field thermal effects governed by the probe size and tip-sample thermal resistance rather than by the modulation frequency. ${ }^{2} \mathrm{~A}$ different approach that avoids this complication is based on the local probing of thermal expansion: subsurface periodic resistive heating $^{5,6}$ or chopped optical radiation ${ }^{7-9}$ generate surface vibrations that can then be mapped to a lateral resolution of a few nanometers in standard atomic force microscope (AFM) or scanning tunneling microscope configurations.

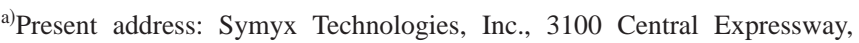
Santa Clara, CA 95051

b) Author to whom correspondence should be addressed; electronic mail: assp@kino-ap.eng.hokudai.ac.jp
}

This produces additional contrast through the thermal expansion coefficient. However, such imaging with these ac local thermal probing techniques so far has been done at frequencies $\$ 100 \mathrm{kHz}$, corresponding to a thermal penetration depth in insulators that is greater than $\sim 1 \mu \mathrm{m}$. This is still too low a frequency for optimal ac thermal imaging of thin films or structures of thickness in the $\sim 100 \mathrm{~nm}$ range or below. There is, therefore, a pressing need to extend such ac local thermal imaging to significantly higher frequencies.

Toward this end, we make use of a technique that we term optical heterodyne force microscopy (OHFM), involving the local probing of photothermally induced ultrasonic surface vibrations. Figure 1 shows the experimental setup, based on a commercial AFM (TM Microscopes, CP-M). We illuminate a region of the sample surface $D \sim 2 \mu \mathrm{m}$ in diameter directly below the tip at normal incidence with $\mathrm{MHz}$ chopped light (at $f_{1}=4.223 \mathrm{MHz}$ ) of typical average power $P=0.7 \mathrm{~mW}$ and wavelength $830 \mathrm{~nm} .{ }^{10}$ We therefore choose a transparent silicon nitride cantilever ${ }^{11}$ (of spring constant $0.1 \mathrm{Nm}^{-1}$ ) and tip (of radius $20 \mathrm{~nm}$ ). In addition to a dc

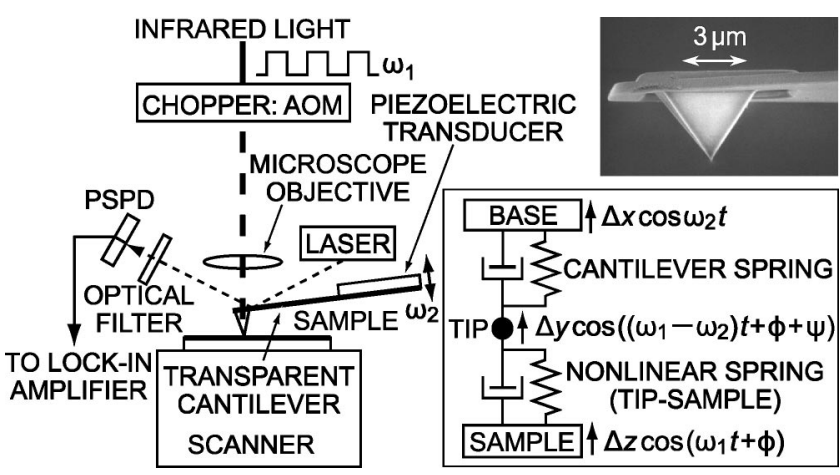

FIG. 1. Schematic diagram of the experimental setup and model used for optical heterodyne force microscopy. PSPD means position-sensitive photodiode and AOM means acousto-optic modulator. In the model, the phase $\psi$ is the part of the phase not related to the phase $\phi$ of the thermal expansion. Also shown is a scanning electron micrograph of the tip. 

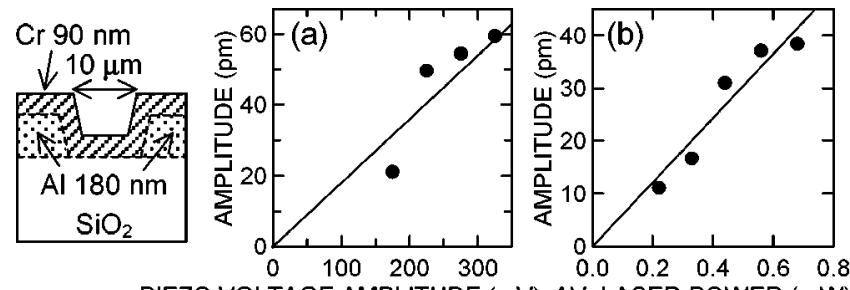

PIEZO VOLTAGE AMPLITUDE (mV) AV. LASER POWER (mW)

FIG. 2. Tip vibration amplitude as a function of (a) the excitation voltage of the piezoelectric transducer at constant average laser incident power $(P$ $=0.7 \mathrm{~mW}$ ), and (b) the average incident laser power at constant piezoelectric drive voltage amplitude $(250 \mathrm{mV})$. The straight lines are guides for the eye. The inset shows part of the microstructure cross section.

temperature rise $<10 \mathrm{~K}$, the sample undergoes periodic temperature variations $\sim 5 \mathrm{~K}$, and the surface vibrates at $f_{1}$ $=\omega_{1 / 2 \pi}$ with an amplitude $\Delta z \sim 10 \mathrm{pm}$ that depends on the local optical, thermal, and mechanical properties of the sample. The cantilever base is vibrated with a piezoelectric transducer operating in thickness resonance at an amplitude $\Delta x \approx 0.8 \mathrm{~nm}$ and at a frequency $f_{2}(=4.220 \mathrm{MHz})$, slightly different from $f_{1}$. This vibration is transmitted down the cantilever, which acts as an acoustic waveguide, and produces a vibration of the tip with a dominant component perpendicular to the sample surface. Because of the nonlinearity of the tip-sample force curve, a vibration $(<100 \mathrm{pm}$ in amplitude) of the contacting tip is induced at a frequency $f_{1}$ $-f_{2}(\sim 3 \mathrm{kHz})$. This is much lower than the fundamental cantilever resonance $(38 \mathrm{kHz})$ but much higher than the response frequency of the AFM feedback loop maintaining the constant average deflection of the cantilever (through sample stage motion). The differential output of the AFM photodiode is fed to a lock-in amplifier tuned to $f_{1}-f_{2}$ to obtain OHFM amplitude and phase images. While scanning, only the sample stage is moved, ensuring a well-defined geometry for sample heating. The principle of OHFM is related to that of ultrasonic force microscopy (UFM). ${ }^{12}$

A cross section of part of the multilayer microstructure sample is shown in Fig. 2. For the fabrication, polystyrene beads of diameter $10 \mu \mathrm{m}$ are sprinkled onto a silica substrate and a $180 \mathrm{~nm}$ layer of polycrystalline $\mathrm{Al}$ is then overlaid by electron-beam deposition. The beads are then removed by washing in distilled water, and a final $90 \mathrm{~nm}$ film of polycrystalline $\mathrm{Cr}$ is deposited in the same way. The thickness of the $\mathrm{Al}$ was measured using $\mathrm{AFM}$ and that of the $\mathrm{Cr}$ using picosecond ultrasonics. ${ }^{13}$ This microstructure provides a good test for the detection of subsurface thermal properties on submicron scales for several reasons: (1) The sample surface is optically and elastically homogeneous, allowing us to study thermal contrast. (The optical absorption depth of $\mathrm{Cr}$ at $830 \mathrm{~nm}$ is $\zeta \approx 15 \mathrm{~nm}$, with optical reflectivity $R=0.63$.) (2) The thermal diffusion length of the silica substrate at $\sim 4$ $\mathrm{MHz}$ is $0.25 \mu \mathrm{m}$, restricting the thermal penetration to a submicron depth. ${ }^{14}$ (3) The thermal expansion coefficient of $\mathrm{Al}$ is $\sim 5$ times larger than that of $\mathrm{Cr}$ and is expected to give noticeable contrast. ${ }^{15}$

We first measured the OHFM signals as a function of incident laser power and ultrasonic excitation voltage on the two-layer portion of the sample. The results for amplitude are shown in Figs. 2(a) and 2(b). Within the experimental error $(\sim \pm 20 \%)$, the amplitudes are proportional to the exciDownloaded 15 Aug 2007 to 133.87.26.100. Redistribution subject
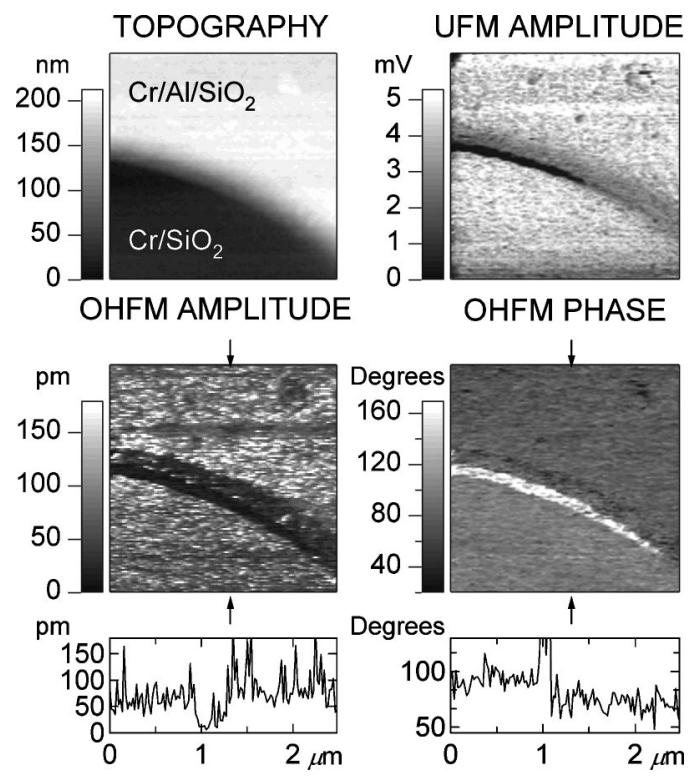

FIG. 3. Topography, UFM, OHFM amplitude, and OHFM phase images for a $2.5 \mu \mathrm{m} \times 2.5 \mu \mathrm{m}$ region of the microstructure. The time for a single line scan of these $128 \times 128$ pixel images is $5 \mathrm{~s}$ for UFM and $3.3 \mathrm{~s}$ for the rest, with a lock-in time constant of $10 \mathrm{~ms}$. The piezoelectric drive voltage amplitudes for UFM and OHFM are $100 \mathrm{mV}$ (maximum) and $250 \mathrm{mV}$, respectively. The scan direction is from the left- to the right-hand side. Cross sections of the OHFM images are also shown (corresponding to the arrows). The optical alignment for OHFM was improved compared to Fig. 2, leading to larger signals here.

tation level in both cases. This behavior is consistent with the predictions of a spring and mass model of the system if the tip-sample interaction is modeled as a nonlinear spring (see Fig. 1 inset). One can show that, for fixed $f_{1}$ and $f_{2}$, the amplitude of the tip at the difference frequency $\Delta y$ $\propto k_{1} \Delta z \Delta x$, where $k_{1}=\partial^{2} F / \partial \delta^{2}$ is the nonlinear coefficient of the force-distance curve. ${ }^{16}$ This implies that OHFM facilitates the measurement of the small sample vibration of amplitude $\Delta z$ by the multiplication with the larger base amplitude $\Delta x$. Another important prediction is that the phase $\phi$ of the vibration at $f_{1}$ is transferred to the phase of the vibration at $f_{1}-f_{2}$. So the OHFM phase directly probes the highfrequency sample response (at $f_{1}$ ).

Figure 3 shows topography, UFM and OHFM amplitude and phase images for a $2.5 \mu \mathrm{m} \times 2.5 \mu \mathrm{m}$ area overlapping the hole (lower region) at an average force $5 \mathrm{nN}$ and with $P=0.7 \mathrm{~mW}$. In both OHFM images the contrast between the two regions is clear: the averaged amplitude ratio $r$ $=\Delta z_{2} / \Delta z_{1} \approx 1.3$, and the averaged phase difference $\Delta \phi$ $=\phi_{1}-\phi_{2} \approx 21^{\circ}$ (where 1 and 2 correspond to the one- and two-layer regions, respectively, and $\phi_{1,2}$ are phase leads). That $r>1$ is expected because of the higher thermal expansion coefficient of Al. The phase difference $\Delta \phi$ was found to be independent, to within the experimental error $\left( \pm 3^{\circ}\right)$, of the excitation conditions over the range probed (see Fig. 2). The halo artifact between the two regions is caused by the slope and curvature of the sample there, ${ }^{12}$ and indicates a lateral resolution of a few nanometers. However, the effective spatial resolution for thermal properties depends on the thermoelastically excited elastic stress distribution and its effect on the surface displacement field.

The UFM image was obtained with the same tip and average force by solely driving the cantilever base with a
to AIP license or copyright, see http://apl.aip.org/apl/copyright.jsp 
sinusoid at $4.2 \mathrm{MHz}$ modulated by a $3 \mathrm{kHz}$ saw-tooth waveform, and by mapping the $3 \mathrm{kHz}$ tip vibration amplitude. ${ }^{12}$ The one- and two-layer regions give approximately equal signals, demonstrating that the surface is elastically homogeneous. ${ }^{17}$ This verifies that the OHFM contrast must be sensitive to differences in subsurface thermal properties.

At $4.2 \mathrm{MHz}$, the thermal penetration into the sample is only $\sim 0.5 \mu \mathrm{m},{ }^{14,15}$ significantly smaller than the optical spot diameter. As a first approximation, we therefore compare our results with a simple quasistatic one-dimensional thermoelastic model. The heat conduction equation, $\kappa \partial^{2} T / \partial z^{2}$ $=c \rho \partial T / \partial t$, is solved subject to the standard interfacial boundary conditions, where $\kappa$ is the thermal conductivity, $c$ is the specific heat, and $\rho$ is the density ( $z>0$ regions). The thermal diffusion length is given by $\mu=\left(2 \kappa / c \rho \omega_{1}\right)^{1 / 2}$. Assuming a surface-localized source, $-\kappa_{1} \partial T / \partial z=I[1+\exp$ $\left.\left(-i \omega_{1} t\right)\right]$ at $z=0$, where $I$ is the average absorbed laser intensity $\left(I \approx P(1-R) / \pi D^{2}\right)$, the surface vibration is derived from $\Delta z \exp \left[-i\left(\omega_{1} t+\phi\right)\right]=\int_{0}^{\infty}([1+\nu(z)] /[1$ $-\nu(z)]) \alpha(z) T(z, t) d z$, where $\alpha(z)$ is the linear thermal expansion coefficient and $\nu(z)$ is Poisson's ratio. Literature values of the required physical parameters are used. ${ }^{14,15,18}$

This allows the calculation of the sensitivity of the OHFM signals to variations in the thermal properties of the buried aluminum layer: We obtain $d \ln \Delta z_{2} / d \ln c=$ $-0.44, d \phi_{2} / d \ln c=5.7^{\circ}, d \ln \Delta z_{2} / d \ln \alpha=0.88, d \phi_{2} / d \ln \alpha$ $=-0.69^{\circ}, \quad$ and $\quad d \ln \Delta z_{2} / d \ln \kappa=-0.001, \quad d \phi_{2} / d \ln \kappa=$ $-0.03^{\circ}$, where $c, \alpha$, and $\kappa$ refer here to aluminum. The unique feature of detection with thermal waves, namely the sensitivity to $c$, is therefore effective here.

Further, we predict $r \approx(30 \mathrm{pm}) /(4.4 \mathrm{pm})=6.8$ and $\Delta \phi$ $\approx 3.9^{\circ}$. Although these relative values have the same sign as in the experiment, they are significantly different. Possible reasons are; (1) the finite optical penetration depth, (2) the neglect of three-dimensional (3D) heat diffusion, (3) the neglect of thermal boundary resistances, and (4) heating effects in the tip itself. We estimate that optical penetration has a negligible influence since $\zeta \ll \mu$. Preliminary estimates ${ }^{19}$ of the effect of 3D heat diffusion on $r$ suggest that this can reduce the amplitude contrast. The effect of thin-film thermal boundary resistance may also play a role. ${ }^{20}$ In addition, thermal expansion of the tip may contribute in general, although simple arguments suggest that this effect is not significant in the present case. ${ }^{21}$

The transition in the OHFM signals between regions of different subsurface structure is remarkably sharp (compared to $\mu$ ), $\sim 50 \mathrm{~nm}$. When the optical excitation overlaps the two regions, a 3D numerical analysis of the thermoelastic problem is clearly necessary to understand this lateral variation. This is beyond the scope of this letter. However, the length scale responsible for these abrupt transitions is likely to be strongly influenced here by the relatively small vertical extent of the buried inhomogeneous region (compared to $\mu$ ). In addition, the change in surface topography will play a role because it impedes both lateral heat flow and lateral stress transmission. Experiments with a series of related sample geometries should help settle this point.
In conclusion, we have demonstrated that subsurface thermal contrast on the submicron level can be locally mapped with a technique based on megahertz photothermal vibrations. The method in its present form should be invaluable for the nondestructive testing of buried nanostructures such as in integrated circuits. Useful contrast from variations in surface optical properties remains to be explored. Moreover, the potential for depth profiling by scanning the optical chopping frequency up to the $1 \mathrm{GHz}$ range and beyond or by the use of the pulsed optical pump and probe technique should open the way to thermal tomography with thermal waves of wavelengths down to the nanometer level, and aid in the study of the physics of diffusive phonon and electron transport in quantum-confined geometries.

The authors are grateful to Osamu Matsuda, Roberto Li Voti, Katsuhiko Inagaki, and Naomi Kumano for helpful discussions.

${ }^{1}$ A. Majumdar, Annu. Rev. Mater. Sci. 29, 505 (1999).

${ }^{2}$ H. M. Pollock and A. Hammiche, J. Phys. D 34, R23 (2001).

${ }^{3}$ A. Hammiche, D. J. Hourston, H. M. Pollock, M. Reading, and M. Song, J. Vac. Sci. Technol. B 14, 1486 (1996).

${ }^{4}$ O. Probst, S. Grafstrom, J. Fritz, S. Dey, J. Kowalski, R. Neumann, M. Wortge, and G. zu Putlitz, Appl. Phys. A: Solids Surf. 59, 109 (1994).

${ }^{5}$ J. Varesi and A. Majumdar, Appl. Phys. Lett. 72, 37 (1998).

${ }^{6}$ J. Bolte, F. Niebisch, J. Pelzl, P. Stelmaszyk, and A. D. Wieck, J. Appl. Phys. 84, 6917 (1998).

${ }^{7}$ J. M. K. Weaver, L. M. Waltipa, and H. K. Wickramasinghe, Nature (London) 342, 783 (1989).

${ }^{8}$ E. Oesterschulze, M. Stopka, and R. Kassing, Microelectron. Eng. 24, 107 (1994).

${ }^{9}$ M. S. Anderson, Appl. Spectrosc. 54, 349 (2000).

${ }^{10}$ Transmission of the light through the (hollow) tip, of refractive index $\sim 2$, was found not to significantly change the diameter of the (approximately Gaussian) optical spot at the sample relative to that above the tip. We use subpicosecond optical pulses with $82 \mathrm{MHz}$ repetition rate, but we checked this had no influence on the sub-10 MHz dynamics here.

${ }^{11}$ TM Microscopes, Sharp Uncoated Microlever E. It is V shaped, of thickness $0.6 \mu \mathrm{m}$, length $140 \mu \mathrm{m}$, width of a V branch $18 \mu \mathrm{m}$, and is inclined to the sample at $15^{\circ}$

${ }^{12}$ K. Inagaki, O. V. Kolosov, G. A. D. Briggs, and O. B. Wright, Appl. Phys. Lett. 76, 1836 (2000).

${ }^{13}$ D. H. Hurley and O. B. Wright, Opt. Lett. 24, 1305 (1999).

${ }^{14}$ O. B. Wright, R. Li Voti, O. Matsuda, M. C. Larciprete, C. Sibilia, and M. Bertolotti, J. Appl. Phys. 91, 5002 (2002).

${ }^{15}$ American Institute of Physics Handbook, 3rd ed., edited by D. E. Gray (McGraw-Hill, New York, 1972).

${ }^{16} \mathrm{O}$. B. Wright and M. Tomoda (unpublished). $\Delta y$ is also proportional to a term involving stiffness and damping parameters.

${ }^{17}$ The local stress penetration is a few times $a$, the tip-sample contact radius (where $a \sim 1 \mathrm{~nm}$ )

${ }^{18}$ G. S. Kino, Acoustic Waves: Devices, Imaging and Analog Signal Processing (Prentice-Hall, New Jersey, 1987).

${ }^{19}$ Based on the calculated 3D temperature field only. In the $\mathrm{Cr}$ and $\mathrm{Al}$ films used, $\kappa$ may be less than the bulk values assumed.

${ }^{20}$ B. Li, J. P. Roger, L. Pottier, and D. Fourier, J. Appl. Phys. 86, 5314 (1999).

${ }^{21}$ The tip length prevents significant heating of the cantilever by thermal conduction in air: $\mu=1.3 \mu \mathrm{m}$ at $4.2 \mathrm{MHz}$. The thermal expansion of the tip may contribute: For silicon nitride, $\mu \approx 0.3 \mu \mathrm{m}$ at $4.2 \mathrm{MHz}$ and $\alpha$ $\approx 2.2 \times 10^{-6} \mathrm{~K}^{-1}$, leading to an estimated maximum contribution to the vibration amplitude at $f_{1}$ equal to $\mu \alpha \Delta T \sim 3 \mathrm{pm}$, assuming periodic temperature variations equal to that of the sample $(\Delta T \sim 5 \mathrm{~K})$. However, the smaller OHFM signal observed for the one-layer region of the sample, where $\Delta T$ is larger, suggests that tip expansion is not dominant. 